\title{
L'éducation au développement durable: entre injonctions ministérielles et obstacles didactiques
}

\section{Nicole Tutiaux-Guillon et Sylvie Considère}

L'éducation au développement durable (EDD), prescrite en France depuis 2004, est censée sarticuler sur les disciplines existantes au primaire et au secondaire. Or le statut des savoirs et les pratiques usuelles de l'histoire-géographie et des sciences de la vie et de la Terre savèrent en contradiction avec ce qui caractérise l'EDD, telle que l'ont définie et expérimentée de nombreux auteurs. En outre, les enjeux éthiques et civiques de l'EDD sont inégalement pris en charge. L'analyse des projets construits par des enseignants, l'observation de cours, des entretiens et des questionnaires passés auprès d'enseignants débutants confortent ce constat. Elle met aussi en évidence d'autres obstacles et parfois des points d'appui pour introduire l'EDD à l'École.

Les discours sur le développement durable (DD) sont scientifiques, médiatiques ou politiques. Les différents acteurs sont sommés de s'impliquer ou de prendre position. L'État prend en charge ce qui devient une nouvelle manière de penser nos rapports au monde et/ou les interrogations sur l'avenir qui en résultent. À l'École ${ }^{1}$, censée préparer l'avenir par l'instruction et l'éducation des futurs acteurs sociaux, économiques, politiques, le «développement durable» devient objet d'enseignement. La question se pose alors d'articuler un mode de penser et d'agir qui impose de faire avec des situations complexes, des savoirs pas toujours stabilisés, des incertitudes, des interprétations parfois contradictoires, des choix de société... et un mode d'enseignement traditionnellement fondé sur la solidité de connaissances prouvées et vérifiées et sur la simplification. Nous présentons dans cet article les contradictions et les difficultés qui obèrent la mise en place de l'éducation au développement durable (EDD). Nous prenons appui sur une recherche conduite depuis $2006^{2}$ et qui a permis, outre une réflexion sur les savoirs scolarisables en la matière, une analyse de contenus des textes officiels depuis 2002, et une étude empirique de l'attitude d'enseignants du primaire et du secondaire face à cette nouvelle prescription à partir de questionnaires et d'observations de pratiques. Le fil directeur est celui d'une difficulté à concilier le statut des savoirs relatifs au DD, les implications éthiques ou politiques liées au 
projet d'EDD d'une part, et le fonctionnement des disciplines, voire l'identité professionnelle des enseignants de l'autre.

\section{Les prescriptions françaises}

La prise en compte des problématiques liées à la préservation de la planète figure dans les programmes français depuis plus de trente ans. Chacune des injonctions successives $(1977,2004,2007)$ s'est faite en parallèle avec des programmes qui n’ont pas été modifiés ou l'ont été ultérieurement. En $1977^{3}$ les élèves du primaire et du secondaire ${ }^{4}$ devaient bénéficier d'une formation leur permettant de «saisir les problèmes de l'environnement». En 20045, «l'éducation à l'environnement pour un développement durable» (EEDD) a été généralisée sur les principes d'une intégration dans les enseignements existants, du croisement des disciplines et de l'ouverture vers le débat et la collaboration avec des partenaires non scolaires. Le but était de permettre aux élèves «de se situer dans leur environnement et y agir de manière responsable», «l'environnement» étant l'entrée prescrite. Les connaissances devaient être vues d'un point de vue scientifique et soumises à une critique mettant en évidence choix et responsabilités. Or l'expression «développement durable» était souvent absente des programmes; «l'environnement» même était inégalement mentionné, selon les disciplines et les niveaux. Cela représentait une première phase. La seconde phase a débuté en 2007, dans le contexte de la Décennie des Nations Unies pour l'Éducation en vue du Développement Durable (2005-2014) et de l'accord-cadre de Vilnius de mise en œuvre de la stratégie de la Commission Economique pour l'Europe pour l'éducation au développement durable (2005). Le Ministère parle dès lors d' «éducation au développement durable». La circulaire 6 réaffirme l'importance des croisements disciplinaires appuyés sur les enseignements obligatoires. Dans le «socle commun des connaissances et des compétences» 7 publié à l'été 2006 la thématique apparaît mais les termes oscillent. Assimiler le socle commun, dit ce texte, "c'est être en mesure de comprendre les grands défis de l'humanité, la diversité des cultures et l'universalité des droits de l'homme, la nécessité du développement et les exigences de la protection de la planète». La compétence 3 (culture scientifique) mentionne la connaissance de l'impact des techniques sur l'environnement, l'attitude de responsabilité vis-à-vis de l'environnement, du monde vivant, de la santé. La compétence 5 (culture humaniste) inclut le concept de «développement durable» comme moyen de comprendre la complexité du monde. Ce texte est parallèle aux programmes ${ }^{8}$. Il y a là un paradoxe: le Ministère impose mais, faute d'inventer une discipline scolaire nouvelle, il prend le risque que les savoirs relatifs au DD et la dimension éducative soient dilués dans les disciplines existantes, installées de longue date et dotées de vulgates. Les programmes récents ont cependant marqué une évolution: la géographie de 5 ème (2008) fait de "développement durable» une notion-clé, une problématique, un thème d'étude; les 
programmes de primaire de 2008 affichent des thématiques dédiées à l'EDD, en sciences et en géographie. Il en est de même pour l'enseignement professionnel (Girault \& Sauvé, 2008).

L'hésitation entre «environnement» et "développement durable» persiste. L'environnement étant déjà intégré dans la vulgate des sciences de la vie et de la Terre, les objets d'enseignement qui mettraient mieux en évidence les interrelations complexes entre société, économie, et environnement sont souvent négligés. La circulaire fondatrice de 2004 définit l'environnement comme un «système complexe qui constitue le milieu de vie de l'Homme» et plaide pour une prise en compte, dans les apprentissages scolaires, des différentes échelles de temps et d'espace et de la complexité, appelant une approche systémique qui articule les composantes environnementale, économique, sociale et culturelle (figure 1).

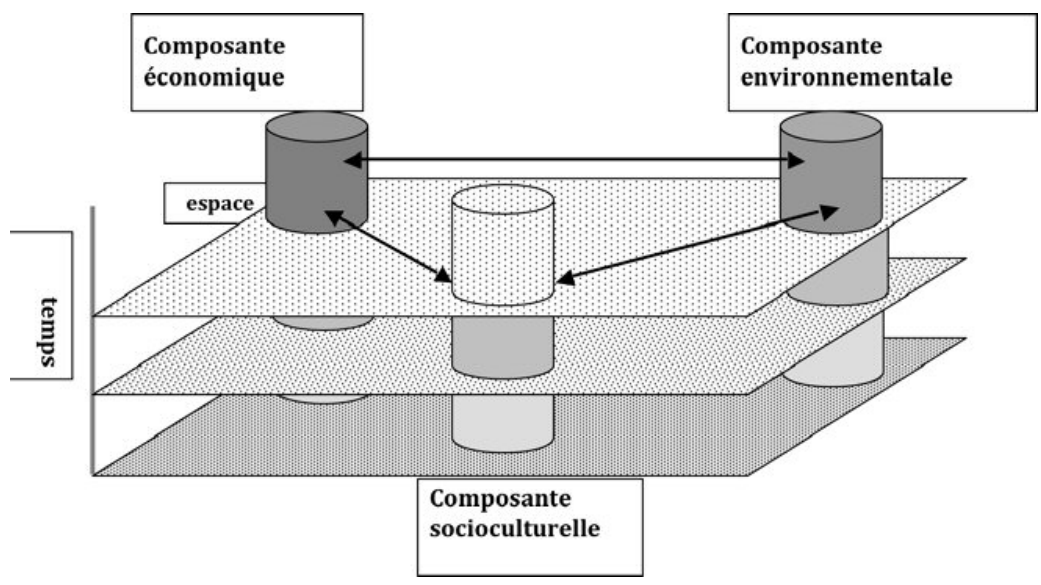

Figure 1. L'environnement de l'homme: un espace, une histoire et des composantes.

Or cette approche recouvre celle qui définit ordinairement le «développement durable», ce qui induit une possible confusion des concepts, ou renvoie le DD au seul statut de projet de société. En outre les définitions développées dans les savoirs de référence sont plurielles, voire divergentes (Mancebo, 2006; Veyret, 2006), et ne correspondent pas toujours avec ces textes officiels, d'où une difficulté pour concilier injonctions et ressources pour enseigner. Pour la recherche que nous menons, nous avons décidé de ne pas normaliser une définition en raison de la multiplicité des approches possibles sur le terrain. Ce choix répondait au souci de laisser les enseignants nous présenter ce que eux entendaient par «développement durable», en particulier dans la classe. Mais nous interrogeons ce qui confere au concept de "développement durable» (comme façon de penser le développement et les relations société-nature) et à l'objet «développement dura- 
ble» (comme projet de développement économique, social et environnemental sous-tendu par des valeurs) un statut particulier au regard d'autres objets enseignés et d'autres finalités de l'enseignement.

Les finalités de l'EDD ont été précisées dans la circulaire de 2007: permettre au citoyen d'opérer ses choix en prenant conscience des responsabilités individuelles et collectives, des solidarités nécessaires entre les territoires et entre les générations. Mais, si les textes officiels de 2004 et 2007 évoquent les engagements politiques de l'État en faveur de l'environnement (la Charte pour l'environnement est un grand principe de la République Française depuis 2005), ils ne font guère référence aux choix politiques qu'ont à faire les citoyens ${ }^{9}$. Les débats, par exemple, y sont pratiques scolaires motivantes et non pratiques de démocratie. L'échelle locale est pointée en 2004 comme celle qui offre des exemples concrets, et en 2007 comme celle où les acteurs peuvent s'impliquer, ce qui motiverait les élèves et les initierait à l'exercice de la citoyenneté ${ }^{10}$. Les échelles nationale, européenne ou régionale, qui constituent les fondements mêmes de la vie politique française, sont absentes. "Citoyenneté/citoyen" n’apparaît qu'une fois dans la circulaire de 2004, trois fois dans celle de 200711, «politique» une fois dans chaque. Les valeurs (équité, précaution, prévention, participation, gestion économe, subsidiarité; voir Pellaud, Giordan \& Eastes, 2007), qui sous-tendent les projets de développement ne sont pas évoquées, hormis la solidarité et la responsabilité. Or leur importance ressort de nombreuses définitions du DD, y compris celle qui sert de référence à la circulaire ministérielle française, et dans les Agendas $21^{12}$. Le «développement durable» est un choix idéologique (Girault \& Sauvé, 2008).

Au terme de ce rapide parcours deux questions se posent. L'une, que nous laissons sans réponse, est celle de l'importance accordée par le Ministère de l'Éducation nationale à l'injonction d'E(E)DD. La valeur contraignante des textes est toute relative; elle dépend de la régulation faite par les inspecteurs et les chefs d'établissement, donc finalement de l'engagement de ces derniers en faveur de l'EDD; elle dépend aussi bien sûr des convictions et des choix didactiques des enseignants. La seconde question les intéresse plus immédiatement: comment intégrer l'EDD dans des disciplines existantes? ${ }^{13}$

\section{Traditions disciplinaires: l'exemple de l'histoire et de la géographie}

En 2005, Boyer et Pommier ont réalisé pour l'INRP une enquête par entretiens auprès d'une cinquantaine d'enseignants du secondaire de disciplines variées, les questionnant sur l'EEDD nouvellement imposée. Les enseignants affirmaient un besoin d'information et un malaise: comment enseigner ce sur quoi les savoirs stabilisés font défaut, ce qui dissimule des enjeux et des opinions, voire ce qui 
n'est qu'effet de mode et d'idéologie (Boyer \& Pommier, 2005)? En filigrane se dessine un modèle d'enseignement que nous présentons ici à grands traits, à partir des recherches sur l'histoire-géographie ${ }^{14}$ dans le secondaire (Tutiaux-Guillon, 2004).

Dans le cours et les manuels, les savoirs déclaratifs et factuels dominent. Les procédures méthodiques d'investigation, les incertitudes, les interprétations sont effacées. Les savoirs présentés par l'enseignant, ceux qu'il valide lors des travaux des élèves, sont donnés comme une vérité suffisante sur le monde et son passé, bien que simplifiée à l'usage des élèves et enseignable en un temps bref (Audigier, Crémieux \& Mousseau, 1996; Clerc, 2002; Tutiaux-Guillon, 2001, 2004). Cette représentation des savoirs, qui pouvait sans écueil s'accorder à une conception positiviste de l'histoire ou de la géographie, subsiste au nom de nécessités institutionnelles et pédagogiques. Les finalités en sont politiques (former un citoyen rationnel, éclairé par la science) et culturelles (transmettre la science et ainsi une culture commune "vraie»). Dans cette conception, ce qui permet aux élèves de dépasser leurs préjugés et de surmonter la fragilité de leur compréhension première, c'est de se voir présenter, de façon convaincante, des connaissances dont la clarté et la validité emportent la conviction (Tutiaux-Guillon, 2009). La circulaire de 2007 affirme la même position: l'École doit apporter des explications s'appuyant sur des connaissances avérées. On vise l'objectivité scientifique. Les disciplines scolaires traitent en classe de faits mis à l'épreuve, de théories et d'interprétations validées.

Sur le plan des approches scientifiques du DD, on a plutôt des hypothèses construites dans des cadres théoriques complexes, et des expérimentations nécessitant un temps dont on ne dispose pas, à compter qu'elles soient possibles. Le concept de «développement durable» est situé dans des paradigmes faisant place à l'incertitude, au désordre, à l'instabilité, à la pluralité des interprétations (Aramburu Ordozgoiti, 1998; Camerini, 2003; Mancebo, 2006). La complexité des relations entre les systèmes sociaux, environnementaux et économiques, à plusieurs échelles de temps et d'espaces, dessine des zones floues, des instabilités, des bifurcations. L'essentiel est moins de viser une connaissance exhaustive du réel que d'en construire des modèles, permettant intelligibilité et décision. Lorsqu'il s'agit de choisir entre ces modèles, ce qui prévaut est leur pertinence selon la situation ou selon le nombre d'alternatives et de voies de recherche. L'École devrait ainsi intégrer des savoirs non stabilisés qui éduquent aussi au doute, présenter différents points de vue pour montrer que différentes pistes de solutions coexistent, autoriser le débat à propos de visions du monde différentes et parfois opposées, affronter des objets complexes, et non les simplifier. Si l'EDD se coule dans le moule des savoirs positifs, certes l'enseignement en est plus aisé - mais au prix d'un dogmatisme épistémologiquement intenable et politiquement douteux (Brunel, 2007; Girault \& Sauvé, 2008; Grumiaux \& Matagne, 2009; Mancebo, 2006). 
En outre, les enseignants hésitent à présenter aux élèves des controverses et des questions socialement vives (Legardez \& Simonneaux, 2006). La visée consensuelle écarte les controverses sociales et la visée scientifique efface les valeurs. Or la question du développement durable est controversée; les approches des chercheurs sont variées, voire contradictoires: développement et environnement peuvent être conciliés ou opposés; le DD peut être vu comme des normes voire une morale ou comme un processus, une dynamique. Le DD peut signer l'abandon du paradigme du progrès ou un nouveau paradigme du progrès centré sur la qualité de la vie (Brunel, 2007; Grumiaux \& Matagne, 2009; Mancebo, 2006; Ricard \& Fortin-Debart, 2004; Veyret, 2006). Quant aux postures sociopolitiques, elles vont du militantisme à la résistance. Mais les textes officiels et les sites institutionnels ne sont guère prolixes sur les controverses autour de la notion de «développement durable»; la multiplicité des définitions est vue comme une faiblesse, une jeunesse à dépasser. L'EDD préconisée est ainsi coupée des débats scientifiques et sociaux. Or les enseignants hésitent à assumer dans la classe autre chose que l'enseignement de savoirs (Clerc, 2002; Legardez \& Simonneaux, 2006; Tutiaux-Guillon, 2001, 2004). Ils redoutent, sur un sujet aussi marqué, d'endoctriner ou d'influencer les élèves. L'enquête de Boyer et Pommier le montre: des enseignants voient l'expression de leur propre position face au DD comme un risque; $20 \%$ dénoncent le catéchisme ou la morale que constituerait l'EDD; la plupart sont réticents à enseigner et évaluer les dimensions éthique et axiologique qui seraient illégitimes car non objectives (Boyer $\&$ Pommier, 2005). Ce refus est enraciné dans une conception de la connaissance scientifique et dans un engagement déontologique. En histoire-géographie, les connaissances disciplinaires sont posées comme les (presque) seules sources légitimes des valeurs démocratiques et des comportements citoyens. Les enseignants de sciences résistent à s'engager dans un domaine politique (Girault \& Sauvé, 2008). La rupture professionnelle exigée est considérable (Aramburu Ordozgoiti, 1998; Mulcahy \& Tutiaux-Guillon, 2005). Enfin, l'approche du DD peut aussi soulever des questions éthiques difficiles, pour des enseignants qui n'ont guère l'expérience de la philosophie (Camerini, 2003; Hernandez \& Albillos, 2004). Les discussions lors des séances de formation à l'IUFM Nord-Pas de Calais (module optionnel $\mathrm{EDD}^{15}$ ) témoignent de cette réticence des enseignants qui attendent seulement savoirs et méthodes pédagogiques.

Dans l'enseignement français de l'histoire-géographie, la mise en activité des élèves est pensée pour les motiver plus que les faire apprendre. Cette représentation se prête fort mal à la réflexion sur l'apprentissage des raisonnements, du jugement, de l'argumentation, de l'approche critique, des valeurs, tous apprentissages associés à l'EDD par les prescriptions et les recherches (de Haan, Mann \& Ried, 2000; Mulcahy \& Tutiaux-Guillon, 2005; Ricard \& Fortin-Debart, 2004; Scott \& Gough, 2003; Tilbury, Stevenson, Fien \& Schreuder, 2002; Wheeler \& Perraca Bijur, 2000). Préconisations et expérimentations en EDD valorisent les 
débats, la résolution de problème, le travail critique, le travail collaboratif, la création individuelle et collective. Ces pratiques ne sont pas seulement posées comme plus efficaces; elles préfigurent aussi des formes d'action sociales estimées favorables au DD. Selon ces travaux, éduquer au DD, c'est former les jeunes à juger en termes scientifiques et éthiques, en termes de probabilités et de risques, et à choisir dans l'incertitude, les former au raisonnement systémique, au raisonnement multiscalaire, à l'analyse des conflits d'intérêts, à la résolution de problèmes, à penser la conjoncture dans le long terme. Cela suppose des pratiques de classe bien davantage centrées sur les modes de penser et d'agir que sur les savoirs à «faire passer». Certaines de ces pratiques sont méconnues des enseignants. D'autres sont rejetées. Par exemple, les enseignants d'histoire-géographie ne voient guère d'intérêt didactique au débat. Il appartiendrait au registre de l'opinion, les élèves n'ayant pas les connaissances et les aptitudes pour y atteindre une certaine vérité. Or le débat entre scientifiques, experts, acteurs semble essentiel à l'EDD, car il correspond à un mode de gouvernance d'une part, de résolution de problèmes d'autre part. Les enseignants mettent aussi en cause les difficultés de leurs élèves: faible niveau, manque de concentration, capacités insuffisantes d'abstraction et d'expression, manque d'implication, même s'ils leur reconnaissent des qualités de curiosité, de générosité et d'altruisme propices à une compréhension des problèmes (Boyer \& Pommier, 2005). L'éducation au DD peut alors sembler un idéal utopique dont les plus réalistes se dispenseraient en se limitant à l'ins-truction. La difficulté est aussi que le DD est plus considéré par les enseignants comme un projet de développement, une façon d'agir dans et sur le monde, que comme un mode de pensée. Les projets mis en ligne sur le site officiel eduscoll ${ }^{16}$ ou construits en formation en témoignent: ce sont les actions et les informations qui y sont explicitées et non les apprentissages intellectuels des élèves, dans le secondaire comme dans le primaire.

\section{Hésitations et difficultés des enseignants}

Ce sont bien au final les enseignants qui choisissent ce qui s'enseigne dans le quotidien des classes, en tenant compte des contraintes de la forme scolaire et du contexte dans lequel ils travaillent, en tenant compte de leur identité professionnelle, de leurs valeurs éducatives, de leur éthique politique et de leur propre conception de l'histoire et de la géographie côté science et côté scolaire. On pourrait aussi ajouter qu'y contribuent les conceptions qu'ont les professeurs de l'apprentissage et de l'éducabilité de leurs élèves, et l'interaction avec ces élèves. Nous nous appuyons ici sur des observations ${ }^{17}$ de professeurs d'école qui souhaitent contribuer à l'EDD (2 classes de CE2 d'une éco-école, 1 classe de CM1 et 1 classe de CM2 de l'agglomération lilloise) et des questionnaires auprès d'enseignants en formation initiale. 


\section{Un objet complexe hors de portée des élèves?}

Certaines pistes de travail posent la question des savoirs à maîtriser. Un travail initié dans une classe de CE2 sur les déchets et leur devenir en donne un exemple, celui des piles (observation mai 2008). Les élèves font l'inventaire des objets que l'on jette. Les piles posent un problème récurrent que l'enseignante doit résoudre avant d'aller plus loin. Pour certains élèves, les piles ne doivent pas être jetées parce que, pleines d'électricité, elles risquent de brûler les poubelles, pour d'autres les magasins dans lesquels on les a rapportées les rechargent ou les fondent, pour l'un d'eux, recharger les piles c'est les remplir de pollution. L'enseignante entend donc travailler les relations entre pile et pollution. La difficulté est d'expliquer aux élèves, documents à l'appui, que les piles contiennent des métaux lourds qui, mis en contact avec la terre ou l'eau, les polluent très longtemps et que si les piles sont incinérées, ces métaux dégagent des gaz toxiques qui contaminent l'atmosphère. Les élèves doivent comprendre des notions comme la dissolution d'un produit dans l'eau, l'expansion d'un gaz dans un autre, la combustion et ses résidus, le cycle de l'eau, savoir que les plantes absorbent l'eau, avoir une idée des quantités et des durées. Ces obstacles peuvent être en partie surmontés grâce à une simplification, mais l'enseignante prend le risque que certains élèves n'aient pas saisi une partie des explications et celui de renforcer des approches biaisées de phénomènes que les élèves retravailleront dans les années ultérieures. Combien d'autres professeurs se limiteront à l'injonction de ne pas jeter les piles à la poubelle parce que c'est dangereux ou écarteront le problème qui exige une maîtrise de savoirs scientifiques?

L'analyse d'une séquence de sciences dans la classe de CM1 et d'affiches produites par les mêmes élèves lors d'une classe transplantée «sciences» quelques mois plus tard, permet de poser des questions complémentaires. Une des affiches traite des économies d'énergie dans une maison. Les textes en sont des injonctions (par exemple «1 - Préférer la douche au bain: 701 au lieu de 200 l»18), mais rien ne distingue autrement cette affiche de celles qui présentent des informations scientifiques ou techniques. Consommer moins semble un mot d'ordre qu'il n'est pas nécessaire de justifier. Les affiches réalisées semblent ainsi correspondre aux traditions scolaires. Il n'y figure ni référence aux valeurs ou à la citoyenneté (ou à l'éco-citoyenneté) ni mention des débats (par exemple pour l'affiche sur les éoliennes). Dans le cours, le «bien pour l'environnement» est opposé au «dangereux». Un statut de vérité est accordé à cette affirmation, comme aux savoirs. La complexité des rapports entre savoirs scientifiques, comportements sociaux et choix de valeurs n'est ni apparente ni démêlée.

Il s'agit là de données sur l'enseignement primaire. Nous n'avons pu observer des séances de classe dans le secondaire. Mais les enseignants, au moins les débutants, témoignent aussi de difficultés. ${ }^{19}$ 


\section{DD et EDD en marge des savoirs disciplinaires?}

Deux modules pluridisciplinaires, associant $31 \mathrm{PLC}^{20}$ de l'enseignement général (histoire-géographie, sciences de la vie et de la Terre [SVT]) et professionnel (lettres-histoire, biochimie, santé et environnement) ont permis le recueil de réponses à un questionnaire sur le DD et l'EDD ${ }^{21}$. Nous en retenons ici ce que nous relions aux hésitations des débutants. Les définitions spontanées du développement durable sont variables; certaines sont proches de la version officielle et de celle de Brundtland (tronquée). Les mots associés (nous en demandions cinq) sont extrêmement dispersés sur l'ensemble des réponses (98 mots différents). L'environnement et l'économie dominent. Le social, les valeurs, le politique sont très faiblement mentionnés. Des actions représentatives sont souvent évoquées, en réponse à différentes questions; des savoirs, des modes de pensée, jamais. Léducation même n'est mentionnée qu'une fois. Ces PLC sont peu au clair sur le $\mathrm{DD}$, et surtout en ont une approche restreinte à une trilogie environnementéconomie-action en faveur de la planète qui est aussi celle que privilégient les médias. Interrogés sur ce qu'ils attendent du module, les réponses sont souvent convergentes: trouver le lien avec les programmes, en savoir plus. Lorsque les formatrices font apparaître les questions épistémologiques, les apprentissages intellectuels, les enjeux éthiques et politiques d'une «éducation à», les difficultés surgissent. Elles ne résident pas dans les insuffisances des connaissances des PLC mais dans leur conception du métier et de leur discipline, dans leur rapport à la prise en charge des valeurs, des savoirs instables, des situations complexes...

Des PLC d'histoire-géographie ont refusé de faire contribuer l'histoire à l'EDD, par crainte de l'anachronisme s'ils posent au passé des questions du présent et par crainte de l'interprétation abusive ${ }^{22}$. La majorité ${ }^{23}$ exprime aussi le souci de traiter d'abord le programme d'histoire, de géographie, d'éducation civique et d'aborder la question du DD seulement s'il reste du temps. La pensée de l'avenir, et sa prise en charge en classe, déconcertent. Un questionnaire comportant huit questions ouvertes a été diffusé auprès des 34 PLC2 de 2008-2009; il n'a reçu que six réponses 24 (!). Les résultats, modestes, confirment les constats déjà mentionnés. Les réponses correspondent à des positions où le DD n'est pas une façon de penser mais un projet de développement. La dimension conceptuelle est rarement perçue; l'articulation avec les modes de pensée l'est encore moins - or tous les répondants se déclarent convaincus de l'importance de l'EDD. Ce qui serait susceptible d'être point d'appui pour l'EDD relève des représentations sociales partagées de l'enseignement de l'histoire-géographie: la formation à l'esprit critique, l'éducation du citoyen. Il s'agit de prémunir les élèves contre le flou ou la simplification outrancière des discours médiatiques. Il s'agit aussi de les responsabiliser et de les aider à faire des choix posés comme politiques.

Le rapport à la discipline, constitutif de l'identité des enseignants du secondaire, peut ainsi se révéler plus un obstacle qu'un point d'appui pour le développement de l'EDD. 


\section{Une autre façon d'enseigner? Les projets interdisciplinaires}

La circulaire de 2004 a donné lieu dans de nombreuses écoles primaires à des travaux officiellement publiés (sur eduscol) en une base de données de 131 projets entrepris entre 2003 et 2005 dont 88 sont résumés par les enseignants qui les ont mis en œuvre. On y trouve les objectifs visés, le ou les niveaux et les disciplines scolaires concernés, et les partenaires éventuels. Les différents résumés sont classés sur le site selon des rubriques que nous avons reprises pour élaborer le graphique (figure 2). Les thèmes relevés appartiennent plutôt aux sciences et celui sur les déchets est plébiscité. Les disciplines de la "culture humaniste» ${ }^{25}$, sont en retrait: le paysage, premier thème par le nombre de projets (14), ne concurrence que le quatrième thème de sciences, et les autres thèmes sont peu nourris.

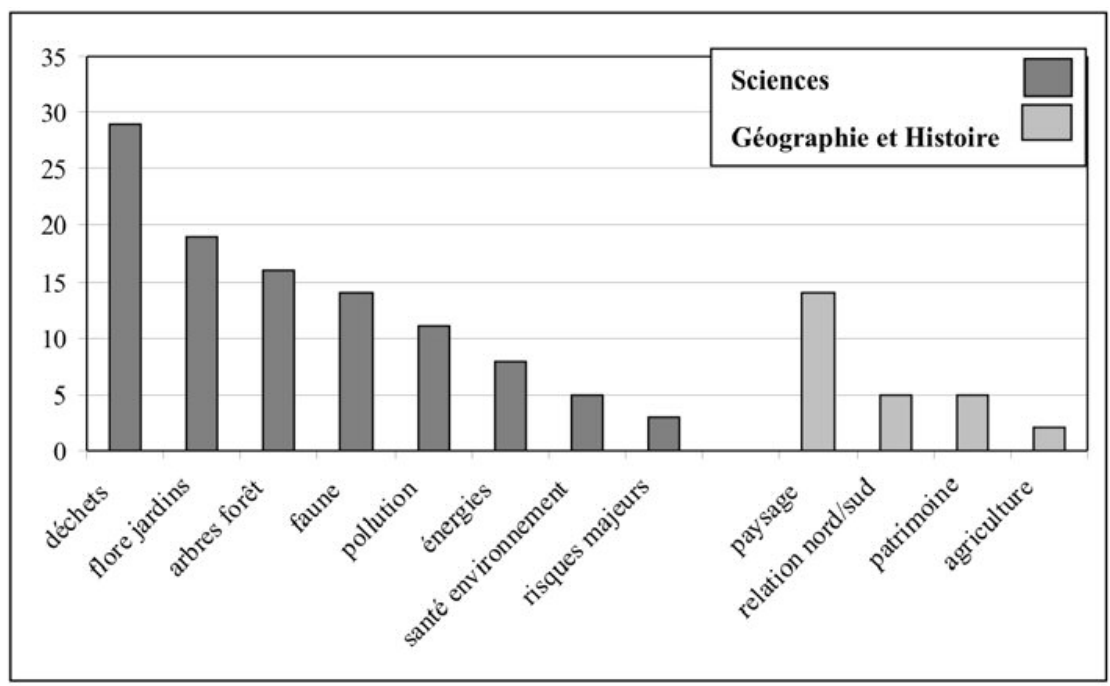

Figure 2. Projets présentés en sciences et en histoire et géographie pour les écoles primaires.

Nous avons analysé dans les résumés disponibles l'architecture du projet, les disciplines concernées, les apprentissages mobilisés et la prise en compte des composantes du DD. Nous avons particulièrement étudié la manière dont le projet était présenté: mise au premier plan d'une réalisation concrète, d'une démarche, d'objectifs d'apprentissages ou de contenus disciplinaires. Ces données présentent des limites: les résumés sont très courts (de 5 à 10 lignes) et les éléments qu'ils contiennent sont déclaratifs. Notre analyse ne porte pas sur les activités réellement proposées aux élèves, activités auxquelles nous n'avons pas accès, mais sur ce corpus dont nous tirons une première typologie en quatre catégories. 


\section{Les réalisations concrètes (31/88)}

L'objectif de ce type de projet est d'amener les élèves à participer à une action concrète, comme planter (14), trier les déchets ou les recycler (8), informer (7). Cet objectif s'accompagne, pour sept projets seulement, de visites ou de travaux en partenariat ${ }^{26}$ qui visent à apporter des savoirs au programme (germination, mesures, schématisations...).

\section{L'acquisition de connaissances (30/88)}

Ces résumés mettent au premier plan un objectif de compréhension, de sensibilisation ou de prise de conscience. Les enseignants articulent les travaux des élèves sur des visites, des recherches. En général, les savoirs de référence semblent être ceux du programme de sciences (découverte d'un écosystème, connaissance des énergies, réseaux alimentaires trophiques). La moitié des projets se centre sur la flore, la faune, l'énergie, le respect de la vie, le cycle de l'eau, comme des leçons ordinaires de sciences. L'autre moitié présente des objectifs plus clairement orientés sur le DD (pollution, bruit, déchets, fabrication et recyclage des plastiques). Huit projets s'appuient sur la mise en place de la démarche d'investigation. La composante dominante, voire exclusive, est l'environnement.

\section{Les projets à facettes (13/88)}

La structure «à facettes» associe une pluralité de disciplines autour d'un objet. Dans les courts résumés présentés, la juxtaposition disciplinaire prime sur l'explicitation de liens entre les contenus et les actions. Il semble que la convergence thématique suffise à faire lien, sans mise en évidence d'une approche systémique. Par exemple la haie bocagère est posée comme un objet d'étude éclairé par différentes disciplines: visites, expériences, exposés permettent l'acquisition de savoirs en SVT, géographie ou arts. On peut penser que les enseignants, polyvalents, partent des disciplines qu'ils ont à enseigner, et cherchent un objet d'étude global susceptible de motiver les élèves, sans viser une problématisation en termes de DD. Mais le même objet d'étude pourrait, dans une étape ultérieure de la réflexion didactique, permettre un réel croisement de disciplines et une approche complexe.

\section{Les problématiques (14/88)}

Ici, la composante sociale est au premier plan, posée par la question des interactions entre l'homme et son milieu. Les dimensions spatiales et temporelles sont souvent présentes. Pour autant les composantes économique et surtout politique sont inégalement travaillées, tout comme la question des choix de valeurs sousjacents aux choix de développement. Mais l'architecture de tels projets est spécifique en ce qu'elle organise une approche de la complexité à partir d'un questionnement. Un autre projet sur la haie l'illustre bien. Il débute par une étude des paysages locaux et le constat que les haies sont nombreuses, d'où la question de savoir d'où viennent ces formations végétales. Les études mobilisent des savoirs 
en géographie, en histoire, en sciences. Prévoir l'implantation d'une haie vient ensuite, et appelle d'autres contenus: choix des graines, germination. La plantation couronne ces travaux; son entretien régulier donne à nouveau lieu à des observations et des apprentissages. Dans ce projet, la situation étudiée replace l'objet «haie» dans son environnement doté d'un espace, d'un temps, de dimensions économiques, sociales et culturelles: la complexité y est explicite et part d'une question posée au monde, et non des disciplines et des contenus prescrits. Dans les travaux problématisés recensés, nous repérons une architecture en cinq étapes, plus ou moins marquées. Nous la formalisons, même si les résumés ne le disent pas ainsi (figure 3). La première étape est primordiale: c'est là que se conçoit l'approche systémique. Si l'objet d'étude a été dès le départ situé dans une réalité globale, un problème est identifié (étape 2), qui résulte de l'analyse de cette réalité globale; il est nécessaire à l'apprentissage mais on sait qu'il est aussi contingent. Les apports disciplinaires que réclame l'étude sont autant de contributions à la compréhension d'un système socio-spatial et/ou socio-scientifique. Il nous semble qu'une telle structure permet des situations plus favorables à l'EDD, en ce qu'elles associent connaissances, réflexion, parfois résolution de problèmes, action suivie, complexité des relations entre les systèmes sociaux, environnementaux et économiques (et relative incertitude), travail collectif et parfois débats. Mais les projets présentés n'explicitent pas une approche politique (limitant souvent la citoyenneté au tri des déchets) et ne semblent pas travailler les valeurs.

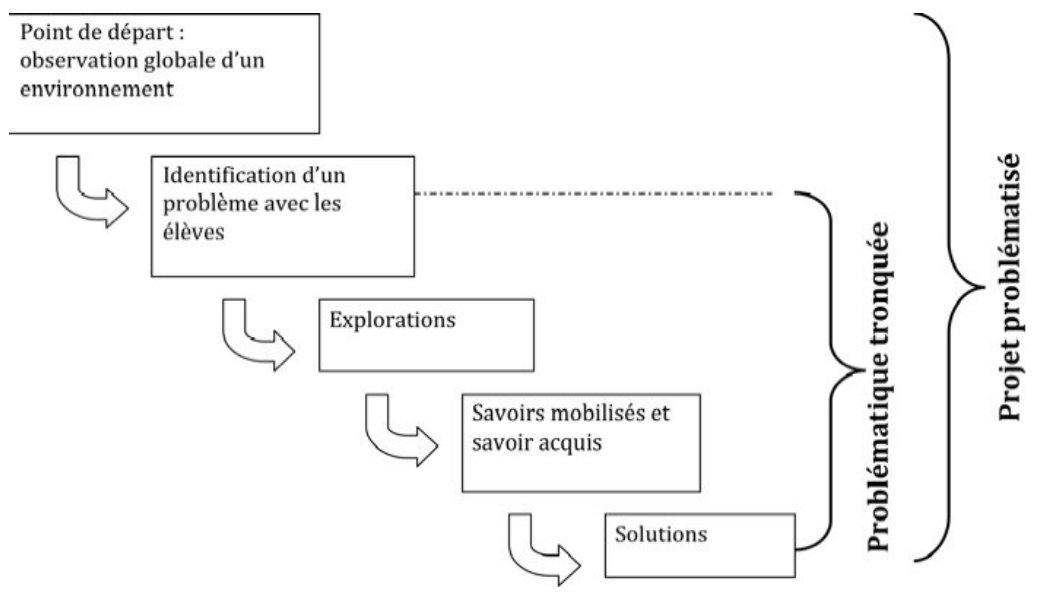

Figure 3. Les étapes de la problématisation d'un projet EDD.

La difficulté, pour les professeurs d'école qui conçoivent ces projets, est de trouver le bon positionnement entre une situation qui mobilise les élèves sur une réalisation et l'acquisition des savoirs au programme. Trouver une solution pour 
qu'un objet d'étude complexe, intéressant et accessible aux élèves, permette aussi qu'ils apprennent des notions prescrites, exige de maîtriser les savoirs généraux et les méthodes propres à chaque discipline et de circonscrire un objet à partir duquel ces savoirs pourront être traités. De telles compétences épistémologiques et didactiques ne sont pas si courantes pour les enseignants du primaire français (Audigier \& Tutiaux-Guillon, 2004).

Les enseignants du secondaire interrogés par Boyer et Pommier (2005) disent eux aussi privilégier pour l'EDD les dispositifs inter- ou pluridisciplinaires ${ }^{27}$, mieux adaptés à la complexité. Sur eduscol, les projets associant diverses disciplines au collège ou au lycée sont nombreux ${ }^{28}$, même si le poids des diverses disciplines y est très inégal. On peut y voir l'amorce d'une structure où le découpage disciplinaire bouge, où une discipline contribue à en éclairer une autre, la géographie par exemple apportant des informations sur l'échelle mondiale d'un problème traité à l'échelle locale en sciences. Sur certains thèmes, hors de toute explicitation, les apports spécifiques de chaque discipline ne se différencient plus ${ }^{29}$. Cependant, à la lecture des projets détaillés accessibles par des liens, il s'agit moins de montrer que chaque discipline problématise différemment les situations que d'afficher une complémentarité, d'éviter les répétitions, et de faire acquérir des savoir-faire transversaux ou des attitudes. Les projets affichent aussi une autre forme de pédagogie, donnant plus de place au travail collaboratif, à l'interrogation collective, à l'autonomie des élèves, y compris pour la problématisation et la recherche documentaire. Là encore les frontières entre disciplines s'effacent, en particulier dans les productions des élèves. Cela suppose chez les enseignants la volonté active de dépasser leurs traditions. Certains estiment d'ailleurs que l'EDD n'entrera dans les faits qu'au prix d'un changement profond de l'organisation scolaire et du travail enseignant (Boyer \& Pommier, 2005).

Lors des modules de formation, nous avons mis l'accent sur une première élaboration de projets interdisciplinaires. C'est cet aspect que la majorité des PLC retient au terme de la formation ${ }^{30}$. Très vite ils rencontrent la difficulté de concilier dans leurs projets des programmes disciplinaires (qui ne sont pas prévus pour permettre des approches croisées), des enjeux de l'EDD et des conceptions des disciplines. Ils peinent à intégrer un travail sur des valeurs ou sur le politique/ civique, un apprentissage de l'incertitude, ou des modes de raisonnement complexes. Un exemple (que nous schématisons dans la figure 4 pour les besoins de l'article) montre cependant la richesse potentielle de leurs projets. Il est réalisé par un groupe associant PLP31 de biologie-santé-environnement, PLP lettres-anglais, PLC de sciences de la vie et de la Terre et PLC d'histoire-géographie. 


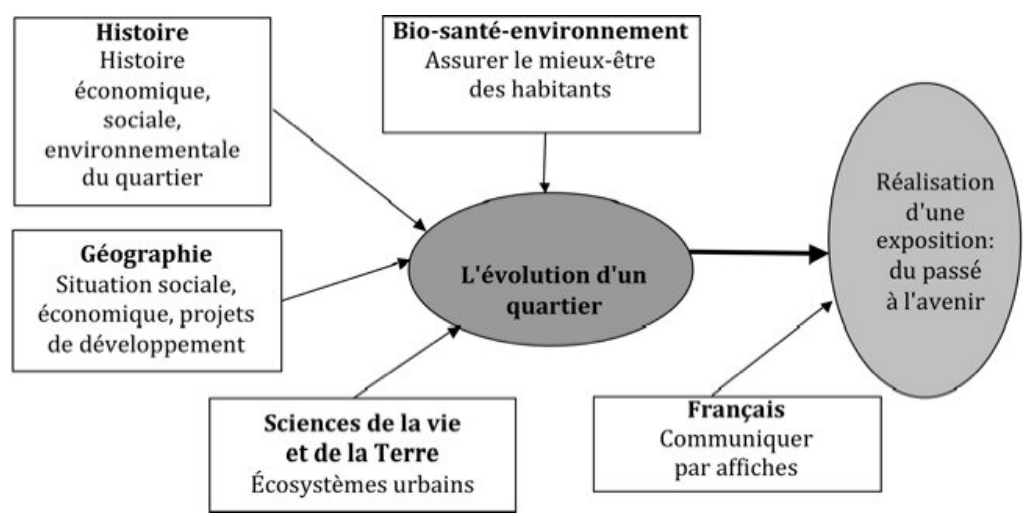

Figure 4. Potentiel interdisciplinaire d'un projet EDD.

Si le français ne contribue pas ici à la réflexion sur le DD mais à ce qui peut et doit se communiquer, les autres disciplines en abordent diverses dimensions, y compris sur une temporalité prenant en compte le passé, le présent et le futur et en incluant l'idée que les données sur lesquelles travaillent les élèves ne permettent pas une prospective sûre. En outre ces PLP/PLC imaginent amener leurs élèves à prévoir différents futurs possibles selon le respect ou non de principes favorables au DD. La complexité est au rendez-vous. La lettre des programmes est un peu malmenée, en particulier dans la perspective d'une programmation annuelle. Mais la dimension éthique ou politique est absente, alors que le thème le permettrait.

Les projets construits dans ce module interdisciplinaire sont surtout des projets «à facettes» et interrogent rarement les valeurs sous-jacentes. Les enseignants découvrent les disciplines des autres, leur temps de réflexion est bref ( 3 à 6 heures) et l'une des contraintes imposées - l'articulation des savoirs disciplinaires et d'une action réalisée par les élèves - est source d'interrogations sur sa propre discipline. Cette expérience recoupe, modestement, les constats présentés par d'autres chercheurs sur les projets interdisciplinaires en EDD (Girault \& Sauvé, 2008).

\section{Conclusion ouverte}

L'EDD est-elle une éducation à la veille critique, à l'éveil de la conscience citoyenne, à la prise de décisions raisonnées dans l'incertitude? Dans ce cas, l'objet d'étude doit certes permettre la prise en compte des composantes environnementales, économiques, socioculturelles, des temporalités et des échelles en une 
approche systémique, mais il échappe à une définition monodisciplinaire. L'élève s'exercerait, en quelque sorte, à une manière de penser le monde qu'il serait à même de mettre en œuvre en tant qu'adulte citoyen. Mais redéfinir les finalités et les structures qui organisent les apprentissages scolaires ne peut qu'entraîner aussi une redéfinition - au moins locale, temporaire - des disciplines. Ou l'EDD est-elle une éducation qui vise l'acquisition de savoirs et la capacité de chacun à mettre en place de «bonnes pratiques pour la sauvegarde de la planète»? Dans ce cas la seule mutation consiste à introduire plus nettement les comportements comme résultat prescrit de l'éducation scolaire, au nom de l'efficacité. Le «socle commun des connaissances et des compétences» (2006), les textes officiels prescrivant l'E(E)DD (2004, 2007), voire les programmes de 2008 s'inscrivent plus ou moins dans cette perspective. Ils semblent préserver l'existant mais mettent les enseignants dans une position peu tenable: les savoirs ne sont ni aisés à choisir dans une profusion contradictoire, ni stabilisés, ni suffisamment proches de la vulgate pour être aisément transposés; les méthodes proposées sont adaptées à une instruction plus qu’à une éducation ou sont inégalement maitrisées; enfin, faisant silence sur les controverses et sur les conflits potentiels de valeurs, les textes font du DD une évidence indiscutable, alors que les enseignants savent qu'il n'en est rien. Choisir un projet interdisciplinaire est sans doute une solution qui permet de concilier à la fois la préservation de l'identité disciplinaire, les disciplines n'en étant affectées qu'à la marge, et les ambitions d'un travail sur un thème complexe appuyé sur des pédagogies différentes du quotidien. Il n'est toutefois pas assuré que les enjeux épistémologiques et politiques du DD et de l'EDD ne soient pas finalement occultés par des enjeux pédagogiques et institutionnels.

\section{Notes}

1 Dans tout l'article, «École» désigne l'institution dans son ensemble; «école» renvoie au niveau primaire.

2 Analyse et expérimentation de situations d'enseignement-apprentissage pour l'éducation à l'environnement pour un développement durable en histoire, géographie et sciences expérimentales: des situations de référence pour la formation. Recherche de l'Université Lille Nord de France (IUFM Nord-Pas de Calais et Laboratoire Théodile-CIREL EA 4354), 2006-2009, associant Michèle Andréani (IUFM Artois), Cora Cohen-Azria (Université Lille 3, ThéodileCIREL), Béatrice Raveillon (IUFM Artois) et les auteures de l'article.

3 Circulaire du 29 août 1977, Bulletin officiel (ci-après B.O.) nº 31 du 8 septembre 1977.

4 En France l'enseignement primaire comprend la maternelle et l'élémentaire; le cycle 3, dernier cycle de l'élémentaire (CE2, CM1, CM2 soit de 8-9 à 10-11 ans), est celui où sont introduites les disciplines. Le secondaire est organisé en "collège» (de la Gème à la 3 ème, soit

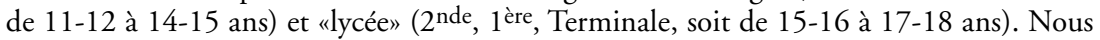
n'évoquons guère ici que l'enseignement général, même s'il existe en parallèle des lycées techniques et professionnels.

5 Circulaire du 8 juillet 2004, B.O. $\mathrm{n}^{\circ} 28,15$ juillet 2004 .

6 Circulaire du 29 mars 2007, B.O. $\mathrm{n}^{\circ} 14,5$ avril 2007.

7 B.O. $\mathrm{n}^{\circ} 29,20$ juillet 2006; au moment où il est publié, ni les programmes du primaire ni ceux du collège ne sont adaptés à ce «socle commun»: il coexiste avec des programmes 
autrement structurés et surtout il affirme des compétences transversales aux disciplines, alors que celles-ci demeurent le mode d'organisation de l'enseignement élémentaire et secondaire. Les expressions en italiques sont soulignées par nous pour cet article.

8 Toutefois les programmes seront modifiés en 2008 pour le primaire et le secondaire.

9 Les thèmes donnés en exemple dans le texte officiel (biodiversité, changements climatiques, ressources non renouvelables) ne sont d'ailleurs pas ceux qui, à première lecture, sembleraient "politiques».

10 Ceci posé, la circulaire suggère des thématiques dont la dimension politique varie: l'éducation aux risques majeurs, l'éducation à la santé ou la promotion de l'utilisation du vélo à l'école...

11 Décomptée la référence à la Déclaration des Droits de l'Homme et du Citoyen.

12 La définition du rapport Brundtland postule que le développement n'est viable qu'en conciliant le respect de l'environnement, l'équité sociale et la rentabilité économique. Lidée même de "futur commun» est à comprendre en termes de solidarité intergénérationnelle et en termes de solidarité nord-sud. La définition présentée dans la circulaire officielle française est tronquée, comme souvent aussi dans les médias français: elle écarte «l'absolue priorité donnée aux besoins des plus démunis» et, partant, l'équité...

13 L'impact des textes officiels sur les pratiques réelles est toutefois douteux. Pour le primaire, Audigier et Tutiaux-Guillon ont mis en évidence le rejet ou l'ignorance de ces textes (2004). Un article récent a montré que le rapport des enseignants avec les documents officiels était complexe et dépendait surtout des instances médiatrices que sont les acteurs du management institutionnel et les collectifs de travail (Piot, 2009).

14 En France l'histoire et la géographie sont enseignées par un même professeur. Nous parlons d'histoire-géographie lorsqu'il s'agit de se référer aux pratiques d'enseignement, aux exigences d'apprentissage et au statut des savoirs. Lorsque nous voulons distinguer les contenus spécifiques, nous parlons d'histoire ou de géographie.

15 Module conçu et conduit par deux chercheures de l'équipe; les enseignants en formation initiale s'y inscrivent sur la base du volontariat. Le module a eu lieu en 2008 (12h), 2009 (6h), 2010 (6h). Propos recueillis sur un cahier de terrain en 2008, 2009, 2010.

16 www.eduscol.fr. Base de données «mise en œuvre de l'éducation au développement durable dans les écoles et établissements». Consultations en 2007, 2008 et 2009. Nous n'analysons pas dans cet article les projets fondés sur le recours à des partenaires extérieurs, souvent des associations. Les données disponibles étant actualisées, le corpus sur lequel nous nous appuyons n'est plus en ligne.

17 Prises de notes des échanges oraux entre enseignants, analyse des objectifs d'apprentissage annoncés, analyse du cours dialogué, des supports et activités proposées aux élèves.

18 Voici la suite: «2 - Ne pas laisser l'eau couler lorsqu'on se brosse les dents. 3 - Préférer le lave-vaisselle à la vaisselle à la main [...]», «1 - Utiliser des ampoules à économies d'énergie (elles consomment 6 fois moins d'énergie). 2 - Ne pas laisser les appareils électriques en veille, il faut les éteindre complètement. 3 - Éteindre les lumières quand on quitte la pièce $[\ldots] »$.

19 Par ailleurs dans l'Académie de Lille, les Inspecteurs ont remarqué le très faible engagement des enseignants du secondaire dans l'EDD.

20 Professeurs de lycée et collège en formation initiale dans un Institut Universitaire de Formation des Maîtres. Nous analysons ici les données de 2008 et 2009.

21 Le questionnaire permet à la fois le recueil de données pour la recherche et le travail initial en formation.

22 Constat en formation disciplinaire et didactique en 2007, 2008, 2009, confirmé par les réponses au questionnaire en 2009, à l'IUFM du Nord-Pas de Calais.

23 Constat fait dans les modules optionnels évoqués et dans les formations didactiques depuis 2007, à raison d'une quarantaine de stagiaires chaque année. 
24 Parce que passé en fin d'année, donc à un moment de désengagement de l'IUFM? Ou parce que la question n'intéressait pas la majorité?

25 Expression empruntée au «socle commun des connaissances et des compétences».

26 La circulaire de 2004 préconisait de privilégier des situations concrètes et des sorties scolaires; celle de 2007 insiste sur les partenariats locaux.

27 En particulier les IDD, itinéraires de découvertes pluridisciplinaires au collège et les TPE, travaux personnels encadrés en lycée. Pour plus de précisions voir le B.O. n 31 du 29 août 2002 et le B.O. $\mathrm{n}^{\circ} 24 \mathrm{du} 28$ juin 2000 . Toutefois ces dispositifs sont en recul.

28 Consultation à partir du site eduscol au cours des années 2007 et 2008 . Les projets de lycée étaient surtout présentés par des établissements professionnels ou techniques. Comme indiqué précédemment ces projets ne sont plus accessibles.

29 Par exemple pour le projet sur la vallée de Chamonix de l'Académie de Dijon, les thèmes affichés (approche géographique, étude géologique et glaciaire, aménagement de la vallée, économie et développement durable, risques naturels) distinguent peu géographie, sciences de la vie et de la Terre et sciences économiques et sociales.

30 Les enseignants ont été questionnés au début du module sur leurs motivations et en fin de module doivent rendre pour l'IUFM une «évaluation» de ce qu'ils ont appris.

31 Professeurs de lycée professionnel.

\section{Références}

Aramburu Ordozgoiti, F. (1998). Los valores ambientales en la educación. In Los valores y la didáctica de las ciencias sociales, IX simposio de didáctica de las ciencias sociales (Actes du symposium, pp. 191-200). Edicions de la Universitat de Lleida.

Audigier, F., Crémieux, C. \& Mousseau, M.-J. (1996). L'enseignement de l'histoire et de la géographie en troisième et en seconde, étude descriptive et comparative. Paris: INRP.

Audigier, F. \& Tutiaux-Guillon, N. (2004). Regards sur l'histoire, la géographie, l'éducation civique à l'école élémentaire. Lyon: INRP.

Bader, B. \& Sauvé, L. (Éd.). (en préparation). L'éducation au développement durable, approches plurielles. Sainte-Foy (Québec): Presses Universitaires de Laval.

Boyer, R. \& Pommier, M. (2005). La généralisation de l'éducation à l'environnement pour un développement durable vue par les enseignants du secondaire (rapport d'enquête). Lyon: INRP. Un fichier pdf peut être téléchargé à partir d'un lien figurant sur la page http://acces.inrp.fr/acces/societe/problematique/enquetesEdd/copy_of_index_html/

Brunel, S. (2007). Le développement durable. Paris: PUF.

Camerini, C. (2003). Les fondements épistémologiques du développement durable, entre physique, philosophie et éthique. Paris: L'Harmattan.

Clerc, P. (2002). La culture scolaire en géographie. Rennes: Presses Universitaires.

De Haan, A. G., Mann, J. \& Ried, A. M. (Éd.). (2000). Educating for sustainability / Former à la durabilité. Frankfurt am Main: Peter Lang.

Girault, Y. \& Sauvé, L. (2008). L'éducation scientifique, l'éducation à l'environnement et l'éducation pour le développement durable. Croisements, enjeux et mouvances. Aster, 46, 7-30.

Grumiaux, F. \& Matagne, P. (Éd.). (2009). Le développement durable sous le regard des sciences (2 volumes). Paris: L'Harmattan.

Hernandez, A. J. \& Albillos, S. (2004). Citizenship education and environmental education today: Thoughts and proposals for its articulation. In A. Ross (Éd.), The experience of citizenship, 6th conference of the CICE thematic network (pp. 140-144). London: CICE.

Lautier, N. (1997). À la rencontre de l'histoire. Villeneuve d'Ascq: Septentrion.

Legardez, A. \& Simonneaux, L. (Éd.). (2006). L'école à l'épreuve de l'actualité. Enseigner les questions vives. Issy-les-Moulineaux: ESF.

Mancebo, F. (2006). Le développement durable. Paris: Armand Colin. 
Mulcahy, C. \& Tutiaux-Guillon, N. (2005). Guidelines on citizenship education for sustainable development. London: CICE.

Pellaud, F., Giordan, A. \& Eastes, E. (2007). Vers de nouveaux paradigmes scolaires. Chemin de Traverse, 5 (Les Amis de Circée [Éd.]).

Piot, T. (2009). Interpréter les prescriptions : Une dimension invisible du travail enseignant. Spirale, 43, 215-226.

Ricard, M. \& Fortin-Debart, C. (Éd.). (2004). Actes du Colloque international sur l'éducation à l'environnement pour un développement durable. Pessac: Institut EGID, Université Michel de Montaigne-Bordeaux 3.

Scott, W. \& Gough, S. (2003). Sustainable development and learning, framing issues. London: Routledge Farmer.

Tilbury, D., Stevenson, R. B., Fien, J. \& Schreuder, D. (Éd.). (2002). Education and sustainability: Responding to the global challenge. Gland: IUCN International Union for Conservation of Nature.

Tutiaux-Guillon, N. (2001). L'enseignement de l'histoire sociale au collège et au lycée (Thèse, 1998, Université de Paris 7 Denis Diderot). Lille: Atelier national de reproduction des thèses.

Tutiaux-Guillon, N. (2004). L'histoire-géographie dans le secondaire, analyses didactiques d'une inertie scolaire. Mémoire pour l'Habilitation à Diriger des Recherches, Université Louis Lumière Lyon 2.

Tutiaux-Guillon, N. (2009). Histoire-géographie et éducation au développement durable: entre modèle disciplinaire et nouvelles exigences. In F. Grumiaux \& P. Matagne (Éd.), Le développement durable sous le regard des sciences (vol. 1, pp. 145-158). Paris: L'Harmattan.

Veyret, Y. (2006). Le développement durable: Approches plurielles. Paris: Hatier.

Wheeler, K. A. \& Perraca Bijur, A. (2000). Education for a sustainable future, a paradigm of hope for the 21st century. New York: Kluwer Academic / Plenum pub.

Mots clés: Éducation au développement durable, disciplines scolaires, savoirs scolaires, finalités, interdisciplinarité, enseignants

\section{Die Bildung für eine nachhaltige Entwicklung: zwischen Ministerbefehlen und didaktischen Hindernissen}

\section{Zusammenfassung}

Bildung für nachhaltige Entwicklung (BNE), in Frankreich seit 2004 verpflichtend, soll mit den vorhandenen Disziplinen der Primar- und Sekundarstufe verbunden werden. Wie sich herausstellte stehen jedoch die Bildungsinhalte und die üblichen Unterrichtspraktiken in Geschichte, Geographie und Mensch und Umwelt in Kontrast zu jenem Fachwissen, welches von zahlreichen Autoren als Bestandteil von BNE definiert und angewendet wird. Zudem legt die Analyse von Projekten, welche durch Lehrpersonen erarbeitet wurden, von Unterrichtsbeobachtungen, sowie Gesprächen mit und Befragungen von Berufseinsteigern und -einsteigerinnen nahe, dass die im Konzept BNE angelegte ethischen und zivilgesellschaftlichen Herausforderungen ungenügend in Betracht gezogen werden. Dieselbe Analyse beleuchtet weitere Schwachpunkte in der Umsetzung von BNE und nennt zugleich Ansatzpunkte für eine Einführung von BNE in der Schule. 
Schlagworte: Bildung für nachhaltige Entwicklung, Schulfach, Bildungsinhalte, Bildungsziel, Interdisziplinarität, Lehrperson

\section{L'educazione allo sviluppo sostenibile: tra ingiunzioni ministeriali e ostacoli didattici}

Riassunto

L'educazione allo sviluppo sostenibile (ESS), obbligatoria in Francia dal 2004, deve essere realizzata a partire dalle materie già presenti nella scuola primaria e secondaria. Ma l'impostazione dei saperi e delle pratiche didattiche correnti della storia, della geografia, delle scienze della vita e di quelle della terra si rivelano essere in contrasto con ciò che caratterizza l'ESS, così come è stata definita e sperimentata da diversi autori. E per di più gli aspetti etici e civici delle ESS vengono affrontati in maniera diseguale, come risulta dall' analisi dei progetti didattici elaborati dagli insegnanti, dall'osservazione delle lezioni, dalle interviste e dai questionari sottoposti agli insegnanti da poco entrati in servizio. Questa analisi mette peraltro in luce, insieme ai molti punti deboli, anche le opportunità che possono favorire lo sviluppo dell'ESS nell'insegnamento scolastico.

Parole chiave: Educazione allo sviluppo sostenibile, materie scolastiche, interdisciplinarità, obiettivi di apprendimento, insegnanti

\section{Education for sustainable development: between ministerial injunctions and didactic impediments}

\section{Summary}

Education for sustainable development (ESD), prescribed in France since 2004, is supposed to be developed through the existing disciplines, in primary and secondary education. But the status of knowledge and the usual practices in History-Geography and in Sciences of Life and of Earth contrast with the characteristics of ESD as defined and experimented by many authors. The ethical and civic aims are unequally taken in charge. This statement is supported by the analysis of ESD plans elaborated by teachers, observations of lessons, interviews and questionnaires answered by novice teachers. The same analysis points at other obstacles and possible support for implementing ESD in schools.

Key words: Education for sustainable development, school disciplines, didactics, ethical and civic aims, interdisciplinary education, teachers 